\title{
Arbitrage and completeness in financial markets with given $\boldsymbol{N}$-dimensional distributions
}

\section{Luciano Campi ${ }^{1, \star}$}

Laboratoire de Probabilités et Modèles Aléatoires, Université Paris 6, France, and Dipartimento di Matematica Pura e Applicata, Università degli Studi di Padova, e-mail: campi@ccr.jussieu.fr

Received: date / Revised version: date

\begin{abstract}
In this paper, we focus on the following problem: given a financial market, modelled by a process $S=\left(S_{t}\right)_{t \in \mathcal{T}}$, and a family $\mathbf{M}_{N}=\left\{\mu_{t_{1}, \ldots, t_{N}}: t_{1}, \ldots, t_{N} \in \mathcal{T}\right\}$ of probability measures on $\mathcal{B}\left(\mathbb{R}^{N}\right)$, with $N$ a positive integer and $\mathcal{T}$ the time space, we search for financially meaningful conditions which are equivalent to the existence and uniqueness of an equivalent (local) martingale measure (EMM) $Q$ such that the price process $S$ has under $Q$ the pre-specified finitedimensional distributions of order $N$ (abbr. $N$-dds) $\mathbf{M}_{N}$. We name these two equivalent properties, respectively, $N$-mixed no free lunch and market $N$-completeness. They are based on a classification of contingent claims with respect to their path-dependence on $S$ and on the related notion of $N$-mixed strategy.

Finally, we apply this approach to the Black-Scholes model with jumps, by showing a uniqueness result for its equivalent martingale measures set.
\end{abstract}

Journal of Economic Literature Classification Number : G12, D52

Mathematics Subject Classification (2000): 60G48, 91B28

I wish to thank Marc Yor for helpful suggestions and remarks.

* Present address: Vienna University of Technology, Financial and Actuarial Mathematics, Wiedner Hauptstrasse 8-10/105-1, A-1040 Vienna, Austria 


\section{Introduction}

In this paper we are interested in the existence and uniqueness of an equivalent martingale measure (EMM) $Q$ with the following additional property: under $Q$ the finite-dimensional distributions of order $N$ (abbr. $N$-dds) of the price process must belong in a given family of probabilities on $\mathcal{B}\left(\mathbb{R}^{N}\right)$, where $N$ is a fixed non negative integer.

More precisely, given an arbitrage-free and incomplete financial market consisting of a single stock whose price evolution is described by a process $S=\left(S_{t}\right)_{t \in \mathcal{T}}$ (defined on a filtered probability space $\left.\left(\Omega, \mathcal{F},\left(\mathcal{F}_{t}\right), P\right)\right)$ and given a family $\mathbf{M}_{N}=\left\{\mu_{t_{1}, \ldots, t_{N}}: t_{1}, \ldots, t_{N} \in\right.$ $\mathcal{T}\}$ of probability measures on $\left(\mathbb{R}^{N}, \mathcal{B}\left(\mathbb{R}^{N}\right)\right)$, we search for economically meaningful properties which are equivalent to the existence and uniqueness of an EMM $Q$ under which the $N$-dds ( $N$ being fixed) of $S$ are precisely given by $\mathbf{M}_{N}$.

Such a problem is motivated by the following remark: suppose that the European call prices have arisen as the expected pay-off under a EMM $Q$ in the financial market $S$, where the instantaneous interest rate $r_{t}$ is a deterministic function of time, that is we can represent the price $C(k, t)$ of a call with strike price $k$ and maturity $t$ as

$$
C(k, t)=\beta_{t} \mathbf{E}_{Q}\left[\left(S_{t}-k\right)^{+}\right],
$$

where $\beta_{t}:=\exp \left(-\int_{0}^{t} r_{s} d s\right)$ is a discounting factor, e.g. the current price (time 0 ) of a zero-coupon bond with maturity $t$. When we take a right-derivative with respect to $k$ we find that (see Breeden and Litzenberger (1978) or Dupire (1997))

$$
\frac{\partial}{\partial k+} C(k, t)=-\beta_{t} Q\left(S_{t}>k\right) .
$$

Thus from call prices it is possible to infer the $Q$-distribution of $S_{t}$ for all $t$.

When the instantaneous interest rate $r_{t}$ is random, the above formula still holds, but to obtain from it the law of each $S_{t}$ under $Q$ the agent has to know now calls and zero-coupon bonds prices.

Obviously, behind this reasoning there is the assumption that on the market there exists a family of zero-coupon bonds and call options traded with all maturities $t \in \mathcal{T}$, and (for call options) all potential strike prices $k$. This assumption seems to be quite realistic. In fact many authors (see e.g. Dupire (1997) or Hobson (1998)) observe that call options market is now so liquid that one can realistic treat calls no longer as derivatives but as primary assets, whose prices are fixed exogeneously by market sentiments. 
This viewpoint drives us to interpret $\mathbf{M}_{J, N}$ as a family of exogeneous measures, obtained by the observation of market prices.

From this discussion we can also argue, at least theoretically, that when the given financial market is incomplete, it would be possible to reduce (even considerably) the set of EMMs, by considering only those that match these $N$-dds.

This approach could facilitate even considerably the choice of the "good" EMM. Furthermore, if the set of EMMs matching the $N$-dds inferred from the market is still infinite, one could even apply the various criteria which have been developed in, e.g., Delbaen and Schachermayer (1996) (variance-optimal martingale measure), Föllmer and Schweizer (1991) (minimal martingale measure) and Frittelli (2000) (minimal entropy martingale measure, see also Miyahara (1996)). This would provide a kind of mixed approach in order to select the "right" EMM: use first some "objective" additional information on the distribution of the stock inferred from the market prices and then one of the above "subjective" criteria. Some authors already begin to explore this research field, e.g. see Goll and Ruschendorf (2002) for minimal distance martingale measures, Tierbach (2002) for the mean-variance hedging approach and the book by Föllmer and Schied (2002) (pp. 298-308) for the superhedging approach.

In the financial literature, there exist several articles whose topics are closely related with ours. For instance, Hobson (1998) finds bounds on the prices of exotic derivatives (in particular, lookback options), in terms of the market prices of call options. This is achieved without making explicit assumptions about the dynamics of the price process of the underlying asset, but rather by inferring information about the potential distribution of asset prices from the call prices. Also, quite connected to this approach is the article by Madan and Yor (2002), that contains three explicit constructions of martingales that all have the Markov property and pre-specified marginal densities (see also Carr, Geman, Madan and Yor (2003)). On the other hand, Brigo and Mercurio (2000) construct stock-price processes with the same marginal lognormal law as that of geometric brownian motion and also with the same transition density between two instants in a given discrete-time grid.

The main difference between this part of the literature and our approach is that we fixed also the market model and not only the $N$-dds and, to avoid trivialities, we assume that the market under consideration is incomplete.

The remainder of this paper is structured as follows. Section 2 contains the fundamental theorems of asset pricing (abbr. FTAP) 
with given $N$-dds in a market model, where the underlying probability space is finite; we find that the existence of a EMM with given $N$-dds is equivalent to a property of no-arbitrage, which is stronger than the usual one (see Harrison and Pliska (1981) or Schachermayer (2003)) in the sense that it even allows to trade (statically) in some non-replicable contingent claims. On the other hand, the uniqueness of such a measure is equivalent to the replicability of all contingent claims by a dynamic strategy in $S$ and a static strategy in contingent claims depending on at most $N$ time-coordinates of the underlying price process, i.e. a weaker market completeness condition.

Section 3 presents the FTAP in a market with finite horizon and one risky asset, whose price dynamics is modelled by a continuoustime, real-valued and locally bounded semimartingale.

Finally, in Subsection 3.4 we apply our approach to the BlackScholes model with jumps and we find that, given a family of marginals such that there exists a EMM matching them, and under some standard assumptions on the coefficients, the subset of all such EMMs belonging to $\Upsilon^{1}$ reduces to a singleton.

\section{The finite case}

\subsection{The model}

Let $(\Omega, \mathcal{F}, P)$ be a finite probability space with a filtration $\mathbb{F}=\left\{\mathcal{F}_{t}\right.$ : $t \in \mathcal{T}\}$ where $\mathcal{T}=\{0, \ldots, T\}$ for $T$ positive integer chosen as a fixed finite horizon, i.e.

$$
\Omega=\left\{\omega_{1}, \omega_{2}, \ldots, \omega_{K}\right\}
$$

is a finite set, the $\sigma$-algebra $\mathcal{F}$ is the power set of $\Omega$ and we may assume without loss of generality that $P$ assigns strictly positive value to all $\omega \in \Omega$. We also assume that $\mathcal{F}_{0}$ is trivial and $\mathcal{F}_{T}=\mathcal{F}$.

We consider a financial market with $d \geq 1$ risky assets modelled by an $\mathbb{R}^{d+1}$-valued stochastic process $S=\left(S_{t}\right)_{t \in \mathcal{T}}=\left(S_{t}^{0}, S_{t}^{1}, \ldots, S_{t}^{d}\right)_{t \in \mathcal{T}}$ based on and adapted to the filtered stochastic base $\left(\Omega, \mathcal{F},\left(\mathcal{F}_{t}\right)_{t \in \mathcal{T}}, P\right)$. We shall assume that the cash account $S^{0}$ satisfies $S_{t}^{0} \equiv 1$ for all $t \in \mathcal{T}$. As usual, this means that the stock prices are expressed in discounted terms.

We denote by $\mathcal{H}$ the set of trading strategies for the financial market $S$, i.e. the set of all $\mathbb{R}^{d}$-valued stochastic processes $H=\left(H_{t}\right)_{t \in \mathcal{T}}=$ $\left(H_{t}^{1}, \ldots, H_{t}^{d}\right)_{t \in \mathcal{T}}$ which are predictable with respect to the given filtration, i.e. each $H_{t}$ is $\mathcal{F}_{t-1}$-measurable.

\footnotetext{
${ }^{1} \Upsilon$ is the subset of all equivalent martingale measures $Q^{h}$ corresponding to some parameter $h$ deterministic function of time (see 2.3.4).
} 
We then define the stochastic integral $(H \cdot S)$ as the real valued process $\left((H \cdot S)_{t}\right)_{t \in \mathcal{T}}$ given by $(H \cdot S)_{0}=0$ and

$$
(H \cdot S)_{t}=\sum_{j=1}^{t} H_{j} \Delta S_{j}, \quad t=1, \ldots, T,
$$

where $\Delta S_{t}=S_{t}-S_{t-1}$.

We observe that, having defined the zero coordinate $S^{0}$ of $S$ to be identically equal to 1 so that $\Delta S_{t}^{0} \equiv 0$, this coordinate do not contribute to the stochastic integral (1).

We denote by $\mathcal{M}^{a}$ (resp. $\mathcal{M}^{e}$ ) the set of absolutely continuous (resp. equivalent) martingale measures, i.e. the set of all $P$-absolutely continuous (resp. $P$-equivalent) probability measures $Q$ on $\mathcal{F}$ such that $S$ is a martingale under $Q$.

Throughout the paper we make the following standing assumption: $\mathcal{M}^{e}$ is not empty and it does not reduce to a singleton, i.e. the market is arbitrage-free and it is not complete.

Let $N$ be a positive integer less than $K$ and let

$$
\mathbf{M}_{N}=\left\{\mu_{t_{1}, \ldots, t_{N}}: t_{i} \in \mathcal{T}, 1 \leq i \leq N\right\}
$$

be a family of probability measures on $\mathcal{B}\left(\mathbb{R}^{N \times d}\right)$.

We let $\mathcal{M}_{N}^{a}$ denote the subset of $\mathcal{M}^{a}$ formed by the probability measures under which the law of every $N$-dimensional vector $\left(S_{t_{1}}, \ldots, S_{t_{N}}\right)$ is precisely $\mu_{t_{1}, \ldots, t_{N}}$, and by

$$
\mathcal{M}_{N}^{e}=\left\{Q \in \mathcal{M}_{N}^{a}: Q \sim P\right\}
$$

we denote the set of all EMMs with $N$-dds in $\mathbf{M}_{N}$, that is the subset of $\mathcal{M}_{N}^{a}$ containing the probability measures that are equivalent to $P$.

Throughout the sequel, we will always suppose the consistency of the set $\mathbf{M}_{N}$ with respect to the martingale property, i.e. that there exists a martingale, on some stochastic base, such that its $N$-dds are in $\mathbf{M}_{N}$.

When $N=1$, a necessary and sufficient condition for this property can be found in Strassen (1965) (see also the book by Föllmer and Schied (2002), pp. 103-111). We quote it without proof.

Theorem 1. (Strassen (1965), Theorem 8, pp. 434-435) Let d a positive integer and $\left(\mu_{n}\right)_{n>0}$ be a sequence of probability measures on the measurable space $\left(\mathbb{R}^{d}, \mathcal{B}\left(\mathbb{R}^{d}\right)\right)$. A necessary and sufficient condition for the existence of a d-dimensional martingale, say $\left(M_{n}\right)_{n \geq 0}=$ 
$\left(M_{n}^{1}, \cdots, M_{n}^{d}\right)_{n \geq 0}$, on some filtered stochastic base, such that the distribution of $M_{n}$ is $\mu_{n}$ for all $n \geq 0$ is that all $\mu_{n}$ have means, i.e. $\int|x| \mu_{n}(d x)<\infty$, and that for any concave function $\psi: \mathbb{R}^{d} \rightarrow \mathbb{R} \mu_{n^{-}}$ integrable for each $n \geq 1$, the sequence $\left(\mu_{n}(\psi)\right)_{n \geq 0}$ is non-increasing (the values of the integrals may be $-\infty$ ).

Remark 1. When $N$ is an arbitrary integer less than $K$, a necessary condition to the existence of a martingale $M$ such that the law of every $N$-dimensional vector $\left(M_{t_{1}}, \ldots, M_{t_{N}}\right)$ is $\mu_{t_{1} \ldots, t_{N}}$ is the following: for every concave function $\phi: \mathbb{R}^{N} \rightarrow \mathbb{R}$, every $N$-uple $\mathbf{t}_{N}=\left(t_{1}, \ldots, t_{N}\right)$ and every $s \in \mathcal{T}$, one must have

$$
\int_{\mathbb{R}^{N}} \phi(\mathbf{x}) \mu_{\mathbf{t}_{N}}\left(d \mathbf{x}_{N}\right) \leq \int_{\mathbb{R}^{N}} \phi\left(\mathbf{x}_{N}\right) \mu_{\mathbf{t}_{N} \wedge s}\left(d \mathbf{x}_{N}\right),
$$

where $\mathbf{t}_{N} \wedge s=\left(t_{1} \wedge s, \ldots, t_{N} \wedge s\right)$ and $\mathbf{x}_{N}=\left(x_{1}, \ldots, x_{N}\right)$. Indeed, it suffices to use the conditional Jensen inequality. Generalizing this to the $d$-dimensional case is not difficult and is left to the reader.

In this paper, we treat only the case $d=1$ (i.e. only one risky asset), the multidimensional case being a straightforward extension.

The following proposition shows that, in order to reduce the set of EMMs and facilitate the choice of the "good" one, fixing a nonempty subset of marginals (i.e. 1-dds) of the price process is not a useless operation, unless the price process is trivial, i.e. almost surely constant.

We work on the canonical space $\Omega=\left\{x_{1}, x_{2}, \ldots, x_{m}\right\}^{\mathcal{T}}\left(x_{i}\right.$ real for all $i=1, \ldots, m$, with $m$ a positive integer such that $m N=K$ ), $\mathcal{F}=\mathcal{P}(\Omega) . S$ will be the coordinate process, that is $S_{t}(\omega)=\omega_{t}$ for all $t \in \mathcal{T}$ and $\omega=\left(\omega_{1}, \omega_{2}, \ldots, \omega_{N}\right) \in \Omega$, and $P$ a probability measure on $\mathcal{F}$. This is the right setting for the application of Strassen's theorem (Theorem 1), stating in particular that under the convexorder condition there exists a martingale measure $Q$ (not necessarily $P$-equivalent) for the coordinate process $S$.

Proposition 1. Let $\mathcal{M}^{e}$ be not empty. The following statements hold:

1. If the process $S$ is a.s. constant under $P$, then for every given family of marginals $\mathbf{M}_{1}=\left(\mu_{t}\right)_{t \in \mathcal{T}}$, either $\mathcal{M}_{1}^{a}=\emptyset$ or $\mathcal{M}_{1}^{a}=\mathcal{M}^{a}$.

2. Let $\mathbf{M}_{1}=\left(\mu_{t}\right)_{t \in \mathcal{T}}$ be a given family of marginals such that $\mathcal{M}_{1}^{a}$ is not empty. If $\mathcal{M}^{a}=\mathcal{M}_{1}^{a}$, then $S$ is P-a.s constant.

Proof. 1. We assume that $S_{t}=c P$-a.s. for some real $c \in\left\{x_{1}, \ldots, x_{m}\right\}$ and for all $t \in \mathcal{T}$. Then, either $\mu_{t} \neq \delta_{c}$ for some $t \in \mathcal{T}$ or $\mu_{t}=\delta_{c}$ for all $t \in \mathcal{T}$. In the first case $\mathcal{M}_{1}^{a}=\emptyset$, in the second one $\mathcal{M}_{1}^{a}=\mathcal{M}^{a}$. 
Indeed, obviously $\mathcal{M}_{1}^{a} \subseteq \mathcal{M}^{a}$, and, on the other hand, if $R$ is in $\mathcal{M}^{a}$ then $S_{t}=c$ for all $t \in \mathcal{T} R$-a.s., which implies $R\left(S_{t} \in d x\right)=\mu_{t}(d x)=$ $\delta_{c}(d x)$ for all $t \in \mathcal{T}$.

2. We assume without loss of generality that $P(\{\omega\})>0$ for all $\omega \in \Omega$, and proceed by contradiction. Let $Q$ be a given measure in $\mathcal{M}^{e} \subseteq \mathcal{M}_{1}^{a}$. If $S$ is not degenerate under $P$, there exists a set $A$ and an instant $t_{0} \in \mathcal{T}$ such that $Q\left(S_{t_{0}} \in A\right)>0$. So, we can define, for every such $A$, on $\mathcal{F}$ a probability measure $Q_{t_{0}, A}(\bullet)=Q\left(\bullet \mid S_{t_{0}} \in A\right)$. The probability $Q_{t_{0}, A}$ is a $P$-absolutely continuous martingale measure for the process $S^{\left(t_{0}\right)}:=\left(S_{t}\right)_{t \geqslant t_{0}}$, i.e. it is an element of $\mathcal{M}^{a}\left(S^{\left(t_{0}\right)}\right)$. We assume now that $\mathcal{M}^{a}\left(S^{\left(t_{0}\right)}\right)=\mathcal{M}_{1}^{a}\left(S^{\left(t_{0}\right)}\right)$. Since $Q \in \mathcal{M}_{1}^{a} \subseteq$ $\mathcal{M}_{1}^{a}\left(S^{\left(t_{0}\right)}\right)$, this assumption implies in particular that

$$
Q\left(S_{t_{0}} \in A, S_{t_{0}} \in B\right)=Q\left(S_{t_{0}} \in A\right) Q\left(S_{t_{0}} \in B\right)
$$

for all $A$ such that $Q\left(S_{t_{0}} \in A\right)>0$ and for all $B$. Thus under $Q S_{t_{0}}$ is independent from itself and so $S_{t_{0}}$ must be degenerate under $Q$ and so even under $P$, which is absurd. To complete the proof, it remains to show that $\mathcal{M}^{a}=\mathcal{M}_{1}^{a}$ implies $\mathcal{M}^{a}\left(S^{\left(t_{0}\right)}\right)=\mathcal{M}_{1}^{a}\left(S^{\left(t_{0}\right)}\right)$ for every instant $t_{0}$. We proceed again by contradiction, by assuming that there exists a probability $Q^{\prime} \in \mathcal{M}^{a}\left(S^{\left(t_{0}\right)}\right) \backslash \mathcal{M}_{1}^{a}\left(S^{\left(t_{0}\right)}\right)$ for some $t_{0} \in \mathcal{T}$. We denote by $\left(\nu_{t}\right)_{t \geqslant t_{0}}$ its family of marginals, that is by assumption different from $\left(\mu_{t}\right)_{t \geqslant t_{0}}$ and satisfies the convex-order condition in Theorem 1. We consider now the following family of marginals:

$$
\nu_{t}^{\prime}:=\nu_{t_{0}} \mathbf{1}_{\left\{0 \leqslant t<t_{0}\right\}}+\nu_{t} \mathbf{1}_{\left\{t \geqslant t_{0}\right\}}, \quad t \in \mathcal{T} .
$$

Also the family $\left(\nu_{t}^{\prime}\right)_{t \in \mathcal{T}}$ satisfies the convex-order condition of Theorem 1, so that there exists, on the canonical space, a probability measure $R$ such that the coordinate process $S$ is an $R$-martingale and, for all $t \in \mathcal{T}, R\left(S_{t} \in d x\right)=\nu_{t}^{\prime}(d x)$. Finally, since the space $\Omega$ is finite, $R$ is $P$-absolutely continuous, that is $R \in \mathcal{M}^{a} \backslash \mathcal{M}_{1}^{a}$. The proof is now complete.

\subsection{Path-dependent contingent claims and $N$-mixed trading strategies}

We identify the set of all contingent claims to the space $L^{0}(P)=$ $L^{0}(\Omega, \mathcal{F}, P)$ of all a.s. finite random variables defined on $(\Omega, \mathcal{F}, P)$, and we introduce a classification of its elements based on the notion of path-dependence, which has been introduced by Peccati (2003) in a slightly different framework. 
In order to formalize this notion, we need to define the following spaces: let $\Pi_{0}$ be the whole real line $\mathbb{R}$ and, for $N \in\{1, \ldots, T\}$, let

$$
\Pi_{N}:=v . s .\left\{\varphi\left(S_{t_{1}}, \ldots, S_{t_{N}}\right): \varphi \in L^{0}\left(\mathbb{R}^{N}\right), t_{1} \leq \ldots \leq t_{N} \in \mathcal{T}\right\}
$$

denote the vector space spanned by all random variables that depend on at most $N$ time-coordinates of the process $S$, where $L^{0}\left(\mathbb{R}^{N}\right)$ is the space of all real-valued Borel-measurable functions defined on $\mathbb{R}^{N}$. Obviously, if $N^{\prime} \leq N$ we have $\Pi_{N^{\prime}} \subseteq \Pi_{N}$.

We say that a contingent claim $f \in L^{0}(P)$ has a path-dependence degree (abbr. pdd) less than or equal to $N \in \mathcal{T}$ if $f \in \Pi_{N}$. Furthermore, we say that $f$ has pdd $N$ if it belongs in $\Pi_{N} \backslash \Pi_{N-1}$.

Example 1. Two examples of contingent claims with pdd equal to 1 are an European call (or put) option with maturity $t$ and strike price $k$, whose pay-off function is $\left(S_{t}-k\right)^{+}$and, assuming the price process $S$ positive, an Asian option paying the mean value obtained by the spot price over any subset $J$ of $\mathcal{T}$, whose pay-off function is $(1 /|J|) \sum_{t \in J} S_{t}(|J|$ being the cardinality of $J)$.

Example 2. More generally, two examples of contingent claims with pdd equal to $N$ are a lookback option calculated over any subset $J$ of $\mathcal{T}$ and with strike price $k$ whose pay-off function is $\left(\sup _{t \in J} S_{t}-k\right)^{+}$, and an asian option with maturity $N$ and strike price $k$ with pay-off $(1 /|J|) \sum_{t \in J}\left(S_{t}-k\right)^{+}$.

Remark 2. We observe that the spaces $\Pi_{N}$ are the analogues, in the finite space case, of the Föllmer-Wu-Yor spaces

$\Pi_{N}(X)=v . s .\left\{f\left(X_{t_{1}}, \ldots, X_{t_{N}}\right): f \in L^{\infty}\left(\mathbb{R}^{N}\right), 0 \leq t_{1}<\ldots<t_{N} \leq T\right\}$, where $X=\left(X_{t}\right)_{t \in[0, T]}$ is a standard Brownian Motion, introduced by Föllmer, Wu and Yor (2000) for the study of weak Brownian Motions (see also Peccati (2003), for their financial interpretation in terms of space-time chaos).

We can now introduce the new notion of $N$-mixed trading strategy.

Definition 1. $A N$-mixed trading strategy is a triplet $(x, H, \psi)$, where $x \in \mathbb{R}$ is an initial investment, $H \in \mathcal{H}$ is a dynamic trading strategy in $S$ and $\psi$ is a contingent claim with pdd less then or equal to $N$.

The denomination "mixed trading strategy" comes from the fact that it is a combination of a dynamic strategy in the underlying and a static strategy in a certain contingent claim (i.e. buy it at $t=0$ and keep it until the end). 
Finally, we define another family of spaces, related to the sets $\Pi_{N}$ and with a clear financial interpretation: for $N=0$ we set

$$
\mathcal{G}_{0}:=\{0\}
$$

and for $N \geq 1$

$$
\mathcal{G}_{N}:=\left\{\psi-\mathbf{E}_{N}[\psi]: \psi \in \Pi_{N}\right\}
$$

where for all contingent claims $\psi=\sum_{i=1}^{p} \varphi_{i}\left(S_{t_{1}^{(i)}}, \ldots, S_{t_{N}^{(i)}}\right) \in \Pi_{N}$ we have denoted

$$
\begin{aligned}
\mathbf{E}_{N}[\psi] & :=\sum_{i=1}^{p} \mu_{t_{1}^{(i)}, \ldots, t_{N}^{(i)}}\left(\varphi_{i}\right) \\
& :=\sum_{i=1}^{p} \int \varphi_{i}\left(x_{1}, \ldots, x_{N}\right) \mu_{t_{1}^{(i)}, \ldots, t_{N}^{(i)}}\left(d x_{1}, \ldots, d x_{N}\right)
\end{aligned}
$$

the price of the contingent claim $\psi$ based on the $N$-dds $\mathbf{M}_{N}$, which can be viewed as the price observed on the market.

The elements of $\mathcal{G}_{N}$ are the gains which an investor can obtain by pursuing the $N$-mixed strategies $(0,0, \psi)$, i.e. by investing statically in the contingent claim $\psi$ at the market price $\mathbf{E}_{N}[\psi]$.

\subsection{The first FTAP with given $N$-dds}

In this subsection we study the problem of the existence of an EMM with given $N$-dds $\mathbf{M}_{N}$ for the financial model previously described. We will find that this is equivalent to a stronger notion of no-arbitrage, involving also the contingent claims with pdd less than or equal to $N$.

Following Schachermayer (2003), we denote by

$$
\mathcal{K}=\left\{(H \cdot S)_{T}: H \in \mathcal{H}\right\}
$$

the set of attainable contingent claims at price zero.

On the other hand, the vector subset of $L^{0}(P)$ defined by

$$
\mathcal{K}_{N}^{0}=\text { v.s. }\left(\mathcal{K} \cup \mathcal{G}_{N}\right)
$$

is called the set of contingent claims $N$-attainable at price zero, i.e. the set of all random variables $f \in \mathcal{K}_{N}^{0}$ of the form

$$
f=(H \cdot S)_{T}+\left(\psi-\mathbf{E}_{N}[\psi]\right)
$$

for some $H \in \mathcal{H}$ and $\psi \in \Pi_{N}$. They are precisely those contingent claims that one may replicate by pursuing some $N$-mixed trading strategy $(0, H, \psi)$. 
We call the cone $\mathcal{C}_{N}$ in $L^{0}(P)$ defined by

$$
\mathcal{C}_{N}=\left\{g \in L^{0}(P): \text { there is } f \in \mathcal{K}_{N}^{0}, f \geq g\right\}
$$

the set of contingent claims $N$-superreplicable at price zero, i.e. the set of all contingent claims $g \in L^{0}(P)$ that an investor may replicate with zero initial investment, by pursuing some $N$-mixed trading strategy $(0, H, \psi)$ and then, eventually, "throwing away money".

Definition 2. A financial market $S$ satisfies the $N$-mixed no-arbitrage condition $(N$-MNA) if

$$
\mathcal{K}_{N}^{0} \cap L_{+}^{0}(P)=\{0\}
$$

or, equivalently,

$$
\mathcal{C}_{N} \cap L_{+}^{0}(P)=\{0\}
$$

where 0 denotes the function identically equal to zero.

The previous definition formalizes a more refined notion of arbitrage: an $N$-mixed arbitrage possibility is a $N$-mixed trading strategy $(0, H, \psi)$, such that the replicated contingent claim

$$
f=(H \cdot S)_{T}+\left(\psi-\mathbf{E}_{N}[\psi]\right)
$$

is non-negative and not identically equal to zero. So, if a financial market does not allow for this type of arbitrage, we say that it satisfies $N$-MNA.

Remark 3. If a market model satisfies $N$-MNA, then it also satisfies $N^{\prime}$-MNA for each $0 \leq N^{\prime} \leq N$, where

$$
\mathbf{M}_{N^{\prime}}=\left\{\mu_{t_{1}, \ldots, t_{N^{\prime}-1}, t_{N^{\prime}}, \ldots, t_{N^{\prime}}} ; t_{1}, \ldots, t_{N^{\prime}} \in \mathcal{T}\right\}
$$

but the converse does not necessarily hold.

Remark 4. By the definition of $\mathcal{G}_{0}$, the condition 0-MNA is the usual condition of no-arbitrage (e.g. see Harrison and Pliska (1981) or Schachermayer (2003)).

The first FTAP establishes the equivalence between the condition of no-arbitrage and the existence of an EMM for the stock price process $S$ (e.g. see Harrison and Pliska (1981) or Schachermayer (2003)). The next theorem generalizes this equivalence to our setting. It claims that the existence condition is equivalent precisely to $N$-MNA. 
Theorem 2. For a financial market $S$ the following are equivalent:

1. $S$ satisfies $N$-MNA,

2. $\mathcal{M}_{N}^{e} \neq \emptyset$.

To prove this result we need the following preliminary lemma:

Lemma 1. For a probability measure $Q$ on $(\Omega, \mathcal{F})$ the following are equivalent:

1. $Q \in \mathcal{M}_{N}^{a}$,

2. $\mathbf{E}_{Q}[f]=0$, for all $f \in \mathcal{K}_{N}^{0}$,

3. $\mathbf{E}_{Q}[g] \leq 0$, for all $g \in \mathcal{C}_{N}$.

Proof. The implication 1. $\Rightarrow 2$. is obvious. On the other hand if 2 . holds we have

$$
\mathbf{E}_{Q}\left[(H \cdot S)_{T}+\varphi\left(S_{t_{1}}, \ldots, S_{t_{n}}\right)-\mu_{t_{1}, \ldots, t_{n}}(\varphi)\right]=0
$$

for $H \in \mathcal{H}, \varphi \in L^{0}\left(\mathbb{R}^{N}\right)$ and $t_{1}, \ldots, t_{N} \in \mathcal{T}$. So, if we take alternatively $H \equiv 0$ or $\varphi \equiv 0$, we obtain, respectively, the martingale and the $N$ dds property of the price process $S$.

The equivalence of 2 . and 3 . is straightforward.

Proof. (of Theorem 2) We use the separation Hahn-Banach Theorem as in the proof of Theorem 2.8, Schachermayer (2003):

$2 . \Rightarrow 1$. If there is some $Q \in \mathcal{M}_{N}^{e}$ then by Lemma 1 we have that

$$
\mathbf{E}_{Q}[g] \leq 0, \quad \text { for } g \in \mathcal{C}_{N} \text {. }
$$

But, if there were some $g \in \mathcal{C}_{N} \cap L_{+}^{0} \backslash\{0\}$, then, since $Q \sim P$, we would have

$$
\mathbf{E}_{Q}[g]>0
$$

a contradiction.

$1 . \Rightarrow 2$. We consider the convex hull of the unit vectors $\left(\mathbf{1}_{\left\{\omega_{i}\right\}}\right)_{i=1}^{K}$ in $L_{+}^{0}(P)$, i.e. the set

$$
\mathcal{P}=\left\{\sum_{i=1}^{K} \lambda_{i} \mathbf{1}_{\left\{\omega_{i}\right\}}: \lambda_{i} \geq 0, \sum_{i=1}^{K} \lambda_{i}=1\right\} .
$$

$\mathcal{P}$ is a convex, compact subset of $L_{+}^{0}(P)$ disjoint from $\mathcal{K}_{N}^{0}$. Hence there exists a random variable $\phi \in L^{0}(P)$ and two constants $\alpha<\beta$ such that

$$
\mathbf{E}[\phi f] \leq \alpha<\beta \leq \mathbf{E}[\phi h] \quad \text { for } f \in \mathcal{K}_{N}^{0} \text { and } h \in \mathcal{P} .
$$

As $\mathcal{K}_{N}^{0}$ is a linear space, we may replace, without loss of generality, $\alpha$ by 0 . Hence, we may normalize $\phi$ such that $\mathbf{E}[\phi]=1$ and, by setting $d Q / d P=\phi$ and thanks to Lemma 1 , it is now easy to verify that $Q \in \mathcal{M}_{N}^{e}$. 


\subsection{The second FTAP with given $N$-dds}

In mathematical finance, one says that a market $S$ is complete if each contingent claim $f \in L^{0}(P)$ can be replicated, with a certain initial investment $x \in \mathbb{R}$, by a predictable (dynamic) trading strategy $H \in \mathcal{H}$, i.e. one can write

$$
f=x+(H \cdot S)_{T} .
$$

Here we introduce a weaker notion of market completeness named $N$-completeness, allowing the agents to invest even in some nonreplicable contingent claims with a certain path-dependence on the stock $S$.

Definition 3. A financial market $S$ is $N$-complete if for each contingent claim $f \in L^{0}(P)$ there exists a $N$-mixed trading strategy $(x, H, \psi)$ such that

$$
f=x+(H \cdot S)_{T}+\left(\psi-\mathbf{E}_{N}[\psi]\right) .
$$

Economically speaking, a financial market $S$ is $N$-complete if every contingent claim may be attained by a combination of an initial investment $x \in \mathbb{R}$, a predictable trading strategy $H \in \mathcal{H}$ and a contingent claim $\psi$ with pdd less than or equal to $N$.

Remark 5. Two easy consequences of the definition are the following:

1. if the market is $N$-complete, then it is $N^{\prime}$-complete for all $0 \leq$ $N \leq N^{\prime}$

2. a market which is $N$-complete is even complete if and only if $\mathcal{G}_{N} \subset \mathcal{K}$.

The second FTAP is a very well-known result which relates, under the assumption of no-arbitrage (which is equivalent to the existence of an EMM), the market completeness and the uniqueness of the EMM, so that the problem of evaluating a contingent claim reduces to take its expected value with respect to this measure.

Here, we state and prove an analogue of this theorem, but with the new notion of $N$-completeness.

Theorem 3. For a financial market $S$ satisfying the condition $N$ MNA the following are equivalent:

1. $\mathcal{M}_{N}^{e}$ consists of a single element $Q$,

2. the market $S$ is $N$-complete. 
Proof. First we remark that $L^{0}(P)=L^{0}(Q)$ for each probability $Q \sim P$. For the implication 2. $\Rightarrow 1$. note that (11) implies that, for elements $Q_{1}, Q_{2} \in \mathcal{M}_{N}^{e}$, we have $\mathbf{E}_{Q_{1}}[f]=\mathbf{E}_{Q_{2}}[f]$ for every $f \in L^{0}(P)$ and so $Q_{1}=Q_{2}$.

For the implication 1. $\Rightarrow 2$., we denote $\mathcal{R}_{N}$ the subspace of all contingent claims $f \in L^{0}(P)$ which may be replicated as in (11) and we proceed by contradiction. By assumption $\mathcal{R}_{N} \varsubsetneqq L^{0}(P)$. So, there exists a contingent claim $g \in L^{0}(P) \backslash\{0\}$ which is orthogonal to $\mathcal{R}_{N}$, and we can define a probability measure $\widetilde{Q} \neq Q$, by setting

$$
\frac{d \widetilde{Q}}{d Q}=1+\frac{g}{2\|g\|_{\infty}}
$$

It is easy to verify that $\widetilde{Q} \in \mathcal{M}_{N}^{e}$.

\section{The continuous-time case}

\subsection{Terminology and definitions}

Let $(\Omega, \mathcal{F}, P)$ be a probability space with a filtration $\mathbb{F}=\left\{\mathcal{F}_{t}: t \in\right.$ $[0, T]\}$ satisfying the usual conditions of right continuity and completeness, where $T>0$ is a fixed finite horizon. We also assume that $\mathcal{F}_{0}$ is trivial and $\mathcal{F}_{T}=\mathcal{F}$. Let $S=\left(S_{t}\right)_{t \in[0, T]}$ be a real-valued, $\mathbb{F}$-adapted, càdlàg, locally bounded semimartingale modelling the discounted price of a risky asset. Furthermore let be given a family of probability measures on $\mathcal{B}\left(\mathbb{R}^{N}\right)$

$$
\mathbf{M}_{N}=\left\{\mu_{t_{1}, \ldots, t_{N}}: t_{1} \leq \ldots \leq t_{N} \in[0, T]\right\}
$$

where $N$ is a fixed positive integer, such that the usual consistency condition with respect to the martingale property holds, i.e. there exists a filtered probability space and a martingale with precisely these laws as $N$-dds (see remark below).

In these sections we will investigate the problem of the existence and uniqueness of an equivalent local martingale measure (ELMM) $Q$ for the price process $S$ such that

$$
Q\left(S_{t_{1}} \in d x_{1}, \ldots, S_{t_{N}} \in d x_{N}\right)=\mu_{t_{1}, \ldots, t_{N}}\left(d x_{1}, \ldots, d x_{N}\right)
$$

for every $t_{1} \leq \ldots \leq t_{N} \in[0, T]$.

In the continuous-time case, a necessary and sufficient condition for the consistency of a $N$-dds family with respect to the martingale property has been obtained by Kellerer (1972) for $N=1$ : 
Theorem 4. (H. G. Kellerer (1972), Theorem 3, p. 120) Let $\left(\mu_{t}\right)_{t \in[0, T]}$ be a family of probability measures on $\mathcal{B}\left(\mathbb{R}^{d}\right)$, with first moment, such that for $s<t \mu_{t}$ dominates $\mu_{s}$ in the convex order, i.e. for each convex function $\phi: \mathbb{R}^{d} \rightarrow \mathbb{R} \mu_{t}$-integrable for each $t \in[0, T]$, we must have

$$
\int_{\mathbb{R}^{d}} \phi d \mu_{t} \geq \int_{\mathbb{R}^{d}} \phi d \mu_{s}
$$

Then there exists a d-dimensional Markov process $\left(M_{t}\right)$ with these marginals for which it is a submartingale. Furthermore if the means are independent of then $\left(M_{t}\right)$ is a martingale.

Remark 6. As a matter of fact, Kellerer (1972) proved the above result in the case $d=1$, but its proof is essentially based on his Theorem 1 which holds for any family $\mu_{t}$ of probability measures and each $\mu_{t}$ is defined on a polish space $E_{t}$.

Remark \%. When $N$ is arbitrary, it is immediate to adapt the necessary condition formulated in Remark 1 to the continuous-time case.

A probability measure $Q$ on $(\Omega, \mathcal{F})$ is called an ELMM for $S$ with $N$-dds $\mathbf{M}_{N}$ if $Q \sim P$ and $S$ is a local martingale under $Q$ such that, for each $N$-uple of instants $t_{1} \leq \ldots \leq t_{N} \in[0, T]$, the law of the vector $\left(S_{t_{1}}, \ldots, S_{t_{N}}\right)$ is given by $\mu_{t_{1}, \ldots, t_{N}}$.

We denote by $\mathcal{M}_{\text {loc }}^{e}$ the set of ELMMs and by $\mathcal{M}_{N, l o c}^{e}$ the subset of $\mathcal{M}_{l o c}^{e}$ containing the ELMMs for $S$ with $N$-dds $\mathbf{M}_{N}$. Moreover, $\mu_{t_{1}, \ldots, t_{N}}(\varphi)$ will frequently denote the integral $\int_{\mathbb{R}^{N}} \varphi(x) \mu_{t_{1}, \ldots, t_{N}}(d x)$, for any bounded measurable function $\varphi: \mathbb{R}^{N} \rightarrow \mathbb{R}$.

As in the finite space case, we can introduce a classification on the space of contingent claims $L^{\infty}(P)=L^{\infty}(\Omega, \mathcal{F}, P)$, based on the notion of path-dependence. We denote by $\Pi_{0}$ the whole real line and for $N \geq 1$, we set

$$
\Pi_{N} \equiv v . s .\left\{\varphi\left(S_{t_{1}}, \ldots, S_{t_{N}}\right): \varphi \in L^{\infty}\left(\mathbb{R}^{N}\right), t_{1} \leq \ldots \leq t_{N} \in[0, T]\right\},
$$

where $L^{\infty}\left(\mathbb{R}^{N}\right)$ is the space of all Borel-measurable essentially bounded functions $\varphi: \mathbb{R}^{N} \rightarrow \mathbb{R}$.

We say that a contingent claim $f \in L^{\infty}(P)$ has a path-dependence degree (abbr. pdd) less than or equal to $N$ if $f \in \Pi_{N}$. On the other hand, we will say that a contingent claim $f \in L^{\infty}(P)$ has a pdd equal to $N$ if $f \in \Pi_{N} \backslash \Pi_{N-1}$. Obviously, if $0 \leq N^{\prime} \leq N$ we have $\Pi_{N^{\prime}} \subseteq \Pi_{N}$.

One can easily adapt the finite-case examples (subsection 2.2) to the continuous-time model. 


\subsection{The first FTAP with given $N$-dds}

In this subsection we obtain an extension of the Kreps-Yan first FTAP (see Theorem 4.7 in Schachermayer (2003), p. 43), which states the equivalence between the no-free lunch condition and the existence of an ELMM for $S$.

We recall that an admissible trading strategy is a predictable, $S$ integrable process $H=\left(H_{t}\right)_{t \in[0, T]}$ such that there exists a constant $a \in \mathbb{R}$ which satisfies $H_{t} \geq-a$ for each $t \in[0, T]$, and such that $(H \cdot S)_{T}$ is bounded. We denote by $\mathcal{A}$ the set of all such strategies.

$\mathcal{A}^{\text {simple }}$ denotes the subset of $\mathcal{A}$ formed by all simple trading strategies $H$, i.e. of the type

$$
H=\sum_{i=1}^{n} h_{i} \mathbf{1}_{]_{i-1}, \tau_{i}\right]},
$$

where $0=\tau_{0} \leq \tau_{1} \leq \ldots \leq \tau_{n} \leq T$ are stopping times such that each stopped process $S^{\tau_{k}}$ is uniformly bounded and $h_{i}$ are $\mathcal{F}_{\tau_{i-1}}{ }^{-}$ measurable bounded real-valued random variables, and $\mathcal{K}$ the set

$$
\mathcal{K}=\left\{(H \cdot S)_{T}: H \in \mathcal{A}^{\text {simple }}\right\}
$$

of all attainable contingent claims at price zero.

Definition 4. $A N$-mixed admissible (simple) trading strategy is a triplet $(x, H, \psi)$, where $x \in \mathbb{R}$ is an initial investment, $H \in \mathcal{A}$ ( $\mathcal{A}^{\text {simple }}$ ) is an admissible (simple) trading strategy in $S$ and $\psi$ is a contingent claim with pdd less then or equal to $N$.

We call the subspace $\mathcal{K}_{N}^{0}$ of $L^{\infty}(P)$ defined by

$$
\mathcal{K}_{N}^{0}:=v . s .\left(\mathcal{K} \cup \mathcal{G}_{N}^{0}\right)
$$

the set of contingent claims $N$-attainable at price zero, where

$$
\mathcal{G}_{N}^{0}=\left\{\psi-\mathbf{E}_{N}[\psi]: \psi \in \Pi_{N}\right\} .
$$

Then, all random variables $f \in \mathcal{K}_{N}^{0}$ are of the form

$$
\begin{aligned}
f & =(H \cdot S)_{T}+\left(\psi-\mathbf{E}_{N}[\psi]\right) \\
& =(H \cdot S)_{T}+\sum_{i=1}^{p}\left[\varphi_{i}\left(S_{t_{1}^{(i)}}, \ldots, S_{t_{N}^{(i)}}\right)-\mu_{t_{1}^{(i)}, \ldots, t_{N}^{(i)}}\left(\varphi_{i}\right)\right]
\end{aligned}
$$

for some $H \in \mathcal{A}^{\text {simple }}, \varphi_{i} \in B\left(\mathbb{R}^{N}\right)$, and $t_{1}^{(i)}, \ldots, t_{N}^{(i)} \in[0, T]$ for $1 \leq$ $i \leq p$, i.e. they are precisely those contingent claims that an economic 
agent may replicate with zero initial investment, by pursuing some simple admissible $N$-mixed trading strategy $(0, H, \psi)$.

On the other hand, the elements of $\mathcal{G}_{N}^{0}$ are the gains which an investor can obtain by buying some contingent claim in $\Pi_{N}$ (i.e. whose pdd is less than or equal to $N$ ) at the price given by $\mathbf{M}_{N}$.

We call the cone $\mathcal{C}_{N}$ in $L^{\infty}(P)$ defined by

$$
\mathcal{C}_{N}:=\left\{g \in L^{\infty}(P): f \geq g \text { for some } f \in \mathcal{K}_{N}^{0}\right\}
$$

the set of contingent claims $N$-superreplicable at price zero, i.e. the set of all contingent claims that may be replicated with zero initial investment, by pursuing some $N$-mixed trading strategy $(0, H, \psi)$ and then, eventually, "throwing away money".

Definition 5. We say that a financial market $S$ satisfies the $N$ mixed no-free lunch condition $(N$-MNFL) if

$$
\overline{\mathcal{C}_{N}} \cap L_{+}^{\infty}(P)=\{0\}
$$

where the closure is taken with respect to the weak $k^{*}$ topology $\sigma\left(L^{\infty}, L^{1}\right)$ of $L^{\infty}(P)$.

By following exactly the same steps as in the proof of the YanKreps Theorem (e.g. see Schachermayer (2001), Theorem 4.7, page 43), we can arrive, without any additional difficulty, to the following result, whose proof is so omitted.

Theorem 5. The following properties are equivalent:

1. $S$ satisfies $N$-MNFL,

2. $\mathcal{M}_{N, l o c}^{e} \neq \emptyset$.

\subsection{The second FTAP with given $N$-dds}

We identify the set of contingent claims with the space $L^{\infty}(P)$ of all essentially bounded random variables and we denote by $\tau$ any topology on this space. We recall that $L^{\infty}$ is invariant under equivalent change of probability.

Definition 6. Let $\tau$ be some topology on $L^{\infty}(P)$. A financial market $S$ is $N$-complete for $\tau$ if the set

$$
\mathcal{K}_{N}:=\left\{x+\mathcal{K}_{N}^{0}: x \in \mathbb{R}\right\}
$$

is dense in $L^{\infty}$ equipped with the topology $\tau$. 
$\mathcal{K}_{N}$ is the set of all essentially bounded contingent claims that can be perfectly replicated by a $N$-mixed admissible strategy $(x, H, \psi)$, where $x$ is the initial investment, $H$ is an admissible (dynamic) strategy in $S$ and $\psi$ is a contingent claim with pdd less or equal to $N$.

Now, we establish an analogue of the second FTAP, with the new notion of $N$-completeness and for two kind of topologies on the set of contingent claims. Recall that, given a subset $C$ of a vector space $E$, an element $x \in E$ is extremal in $C$ if the relation $x=\alpha y_{1}+(1-\alpha) y_{2}$ with $y_{1}, y_{2} \in C$ and $\alpha \in(0,1)$ implies $x=y_{1}=y_{2}$.

Theorem 6. Let $Q \in \mathcal{M}_{N, l o c}^{e}$. The following are equivalent:

1. $Q$ is extremal in $\mathcal{M}_{N, l o c}^{e}$,

2. the market $S$ is $N$-complete for $L^{1}(Q)$-topology.

Proof. It is an easy application of Theorem 1 in Douglas (1964) (see also Naimark (1947)).

Corollary 1. Let $Q \in \mathcal{M}_{N, l o c}^{e}$. If $\mathcal{M}_{N, l o c}^{e}=\{Q\}$, then the market $S$ is $N$-complete for $L^{1}(Q)$-topology.

The previous corollary means that if there exists an ELMM with given $N$-dds $\mathbf{M}_{N}$ and if this measure is unique, then each contingent claim can be approximately replicated (with respect to the topology induced by $\left.L^{1}(Q)\right)$ by a $N$-mixed trading strategy.

At present, we do not know if the converse of Corollary 1 holds true. Nonetheless, we are able to give a partial answer to this problem if we change the topology on the contingent claims set $L^{\infty}$.

To prove the next results, we need the following functional analytical result (for more general results of this type with some applications to finance, see Campi (2001)):

Lemma 2. Let $P$ be a probability measure on $(\Omega, \mathcal{F})$ and let $F$ be a subspace of $L^{\infty}(P)$ containing the unit function $1 . F$ is dense in $\left(L^{\infty}(P), \sigma\left(L^{\infty}, L^{1}\right)\right)$ if and only if every probability measure $Q \ll P$, is an extremal point of the set of all probability measures $R \ll Q$ on $(\Omega, \mathcal{F})$ such that

$$
\int f d R=\int f d Q \quad \text { for all } f \in F .
$$

Proof. See Campi (2001). 
Theorem 7. Let the market $S$ be $N$-complete for the weak* topology. Then, there exists at most one $Q \in \mathcal{M}_{N, l o c}^{e}$.

Proof. Assume that there exists two different probability measures $Q_{0}, Q_{1} \in \mathcal{M}_{N, l o c}^{e}$. Since $\mathcal{M}_{N, l o c}^{e}$ is a convex set, for each $\alpha \in[0,1]$ $Q_{\alpha}=\alpha Q_{1}+(1-\alpha) Q_{0}$ is in $\mathcal{M}_{N, l o c}^{e}$. But, since the market $S$ is $N$-complete for the weak* topology, by Lemma 2 , every $Q_{\alpha}$ must be extremal in the set of all probability measures $R \ll Q_{\alpha}$ on $(\Omega, \mathcal{F})$ such that $\int f d R=\int f d Q$ for all $f \in \mathcal{K}_{N}$. This set containing $\left[Q_{0}, Q_{1}\right]$, we obtain a contradiction.

To have an equivalence in Theorem 7 , we have to consider uniqueness in a measures set larger than $\mathcal{M}_{N, l o c}^{e}$. Thus, if we denote by $\mathcal{M}_{N, l o c}^{s}$ the set of all finite signed measures $\nu \ll P$ on $(\Omega, \mathcal{F})$ such that $\nu(\Omega)=1$ and $\int f d \nu=0$ for every $f \in \mathcal{K}_{N}$, we have the following

Theorem 8. Let $\mathcal{M}_{N, l o c}^{s}$ be nonempty. The following are equivalent:

1. the market $S$ is $N$-complete for the weak* topology;

2. $\mathcal{M}_{N, \text { loc }}^{s}$ is a singleton.

Proof. 2. $\Rightarrow 1$. Given a measure $\nu \in \mathcal{M}_{N, l o c}^{s}$, which exists by assumption, we proceed by contradiction. Assume that the market is not $N$-complete for the weak* topology, thus by Lemma 2 there exists a probability $Q_{\alpha} \ll Q$ such that

$$
Q_{\alpha}=\alpha Q_{1}+(1-\alpha) Q_{2}
$$

where $\alpha \in(0,1)$ and, for each $i=1,2, Q_{i}$ is a probability measure on $(\Omega, \mathcal{F})$ absolutely continuous to $Q$ such that

$$
\int f d Q_{i}=\int f d Q \quad \text { for all } f \in \mathcal{K}_{N} .
$$

Now, consider the measures

$$
\nu_{i}=Q_{i}-Q+\nu
$$

for $i=1,2$. For each $i=1,2, \nu_{i} \in \mathcal{M}_{N, l o c}^{s}$. Furthermore, since $Q_{1} \leq$ $\frac{1}{\alpha} Q_{\alpha}$ and $Q_{2} \leq \frac{1}{1-\alpha} Q_{\alpha}$, we have $Q_{i} \ll Q_{\alpha} \ll Q$ for every $i=1,2$. Then, since $\left|\nu_{i}\right| \leq Q_{i}+Q_{\alpha}+|\nu|$, we have $\left|\nu_{i}\right| \ll Q$ for each $i=1,2$. This shows that $\nu$ is not unique in $\mathcal{M}_{N, \text { loc }}^{s}$ and so 2. implies 1 ..

1 . $\Rightarrow 2$. Proceed by contradiction by supposing that $\mathcal{M}_{N . l o c}^{s} ?$ $\left\{\nu_{1}, \nu_{2}\right\}$, with $\nu_{1} \neq \nu_{2}$. Observe now that, by the definition of $\mathcal{M}_{N, l o c}^{s}$, $\nu_{1}(f)=\nu_{2}(f)=1$ for all $f \in \mathcal{K}_{N}$ and $\nu_{1}(\Omega)=\nu_{2}(\Omega)=1$, that is

$$
\nu_{1}^{+}(f)-\nu_{1}^{-}(f)=\nu_{2}^{+}(f)-\nu_{2}^{-}(f)=1, \quad \text { for all } f \in \mathcal{K}_{N},
$$


and

$$
\nu_{1}^{+}(\Omega)-\nu_{1}^{-}(\Omega)=\nu_{2}^{+}(\Omega)-\nu_{2}^{-}(\Omega)=1,
$$

where $\nu_{i}^{+}$and $\nu_{i}^{-}(i=1,2)$ are, respectively, the positive and the negative part of $\nu_{i}$ in its Hahn-Jordan decomposition. This implies

$$
\nu_{1}^{+}(f)+\nu_{2}^{-}(f)=\nu_{2}^{+}(f)+\nu_{1}^{-}(f), \quad \text { for all } f \in \mathcal{K}_{N},
$$

and

$$
\nu_{1}^{+}(\Omega)+\nu_{2}^{-}(\Omega)=\nu_{2}^{+}(\Omega)+\nu_{1}^{-}(\Omega):=k>0 .
$$

Thus, define the two probability measures $Q_{1}$ and $Q_{2}$ as follows:

$$
Q_{1}=\frac{\nu_{1}^{+}+\nu_{2}^{-}}{k}, \quad Q_{2}=\frac{\nu_{2}^{+}+\nu_{1}^{-}}{k} .
$$

Observe that $Q_{1}=Q_{2}$ on $\mathcal{Y}_{d}$ and define

$$
Q:=\alpha Q_{1}+(1-\alpha) Q_{2}
$$

for some real $\alpha \in(0,1)$. It is straightforward to verify that $Q \ll P$ (since $\left|\nu_{i}\right| \ll P$, for $i=1,2$ ) and that $Q_{1}$ and $Q_{2}$ are absolutely continuous to $Q$. We have so built a probability measure $Q$ absolutely continuous to $P$ that is not extremal in the set of all probability measures $R \ll Q$ on $(\Omega, \mathcal{F})$ such that $\int f d R=\int f d Q$ for all $f \in \mathcal{K}_{N}$. Finally, Lemma 2 applies and gives that 1. $\Rightarrow 2$.

\subsection{An application: the Black-Scholes model with jumps}

Now, as an application of our approach, we will study the BlackScholes model with jumps (BSJ) and we will show that when its coefficients (drift, volatility, intensity and jump size) are time-deterministic functions, it admits at most one EMM with pre-specified marginals (1-dds) in the remarkable subset $\Upsilon$ of EMMs. Our presentation of BSJ is based on the survey article by Runggaldier (2002).

Let $(\Omega, \mathcal{F}, P)$ be a probability space on which are defined a Wiener process $\left(W_{t}\right)_{t \in[0, T]}$ and a Poisson process $\left(N_{t}\right)_{t \in[0, T]}$ with deterministic intensity $\left(\lambda_{t}\right)_{t \in[0, T]}$, so the compensated process $M_{t}=N_{t}-\int_{0}^{t} \lambda_{s} d s$ is a $P$-martingale adapted to the natural filtration of $N$. As usual we will work on the augmented filtration

$$
\mathcal{F}_{t}=\sigma\left(W_{s}, N_{s}: s \leq t\right) \quad t \in[0, T]
$$

jointly generated by the Wiener and Poisson processes. This choice for the underlying filtration implies, via the predictable representation property of the Wiener and the (compensated) Poisson process, that $W$ and $N$ are independent. 
We now suppose that the discounted price process $\left(S_{t}\right)_{t \in[0, T]}$ satisfies the following SDE

$$
d S_{t}=S_{t-}\left[a_{t} d t+\sigma_{t} d W_{t}+\gamma_{t} d N_{t}\right] \quad t \in(0, T]
$$

with $S_{0}>0$ constant and where $a_{t}, \sigma_{t}, \varphi_{t}$ are three time-deterministic functions such that:

$-\int_{0}^{T}\left|a_{t}\right| d t<\infty$

$-\int_{0}^{T} \sigma_{t}^{2} d t<\infty$, and $\quad \sigma_{t}>0$ for all $t \in[0, T]$;

$-\int_{0}^{T}\left|\gamma_{t}\right| d t<\infty$ and $\gamma_{t}>-1$ for all $t \in[0, T]$.

By applying Itô's formula to the process $\ln S_{t}$ we find

$$
S_{t}=S_{0} e^{\int_{0}^{t}\left(a_{s}-\frac{1}{2} \sigma_{s}^{2}\right) d s+\int_{0}^{t} \sigma_{s} d W_{s}+\int_{0}^{t} \ln \left(1+\gamma_{s}\right) d N_{s}} .
$$

It is well-known that this model admits an infinite number of EMMs $Q^{h}$, depending on a parameter $h=\left(h_{t}\right)$ and which can be represented as

$$
\frac{d Q^{h}}{d P}=e^{\int_{0}^{T}\left(\lambda_{t}\left(1-h_{t}\right)-\frac{1}{2} \vartheta_{t}^{2}\right) d t+\int_{0}^{T} \vartheta_{t} d W_{t}+\int_{0}^{T} \ln h_{t} d N_{t}}
$$

for any couple of predictable processes $(h, \vartheta)$ where $h_{t} \geq 0$ is arbitrary and

$$
\vartheta_{t}=\sigma_{t}^{-1}\left(-a_{t}-h_{t} \lambda_{t} \gamma_{t}\right) .
$$

Then, under the EMM $Q^{h}$ the dynamic of the price process $\left(S_{t}\right)_{t \in[0, T]}$ is given by

$$
d S_{t}=S_{t-}\left[\sigma_{t} d \widetilde{W}_{t}^{h}+\gamma_{t} d \widetilde{M}_{t}^{h}\right]
$$

where for every $t \in[0, T]$

$$
\widetilde{W}_{t}^{h}:=W_{t}-\int_{0}^{t} \vartheta_{s} d s
$$

and

$$
\widetilde{M}_{t}^{h}:=N_{t}-\int_{0}^{t} h_{s} \lambda_{s} d s
$$

are, respectively, a Wiener process and a compensated Poisson process under $Q^{h}$. Moreover, we denote $\Upsilon$ the set of all equivalent martingale measures $Q^{h}$ corresponding to some parameter $h=\left(h_{t}\right)$ deterministic function of time. 
Remark 8 . We point out that $\Upsilon$ is a very remarkable set, because it contains the pricing measure used by Merton (1976) and also the main equivalent martingale measures investigated by many authors in the incomplete markets literature: the Föllmer-Schweizer minimal martingale measure, the Frittelli-Miyahara minimal entropy martingale measure and the Esscher transform martingale measure (see the paper by Henderson and Hobson (2001)).

We are now able to study the set $\Upsilon\left(\mathbf{M}_{1}\right)$ of EMMs belonging to $\Upsilon$ with given marginals $\mathbf{M}_{1}=\left(\mu_{t}\right)$. The main result is the following:

Proposition 2. Let $\mathbf{M}_{1}=\left\{\mu_{t}: t \in[0, T]\right\}$ be a family of marginals for the price process $S$ such that $\Upsilon\left(\mathbf{M}_{1}\right)$ is not empty. Under our assumptions on the coefficients of the model and if $\lambda_{t}>0$ for all $t \in[0, T]$, there exists only one EMM $Q \in \Upsilon$ for $S$ such that for all $t \in[0, T] Q\left(S_{t} \in d x\right)=\mu_{t}(d x)$.

Furthermore we have the following formulae for $h=\left(h_{t}\right)$ : if $n \in$ $\mathbf{Z} \backslash\{0\}$ and $\left(1+\gamma_{t}\right)^{n}-(n-1) \gamma_{t}-1 \neq 0$ then

$$
h_{t}=\frac{\frac{d}{d t} m_{t}^{(n)}-\frac{\sigma_{t}^{2}}{2} n(n-1) m_{t}^{(n)}}{\lambda_{t}\left[\left(1+\gamma_{t}\right)^{n}-(n-1) \gamma_{t}-1\right] m_{t}^{(n)}},
$$

where for $t \in[0, T]$,

$$
m_{t}^{(n)}=\int_{\mathbb{R}} x^{n} \mu_{t}(d x) .
$$

Proof. Letting $f \in C^{2}(\mathbb{R})$, we apply Itô's formula for discontinuous semimartingales to $f\left(S_{t}\right)$ and obtain

$$
\begin{aligned}
f\left(S_{t}\right)= & f\left(S_{0}\right)+\int_{0}^{t} f^{\prime}\left(S_{s}\right) S_{s} a_{s} d s \\
& +\int_{0}^{t} f^{\prime}\left(S_{s}\right) S_{s-} \sigma_{s} d W_{s}+\frac{1}{2} \int_{0}^{t} f^{\prime \prime}\left(S_{s}\right) \sigma_{s}^{2} S_{s}^{2} d s \\
& +\int_{0}^{t}\left[f\left(S_{s-}\left(1+\gamma_{s}\right)\right)-f\left(S_{s-}\right)\right] d N_{s} .
\end{aligned}
$$

By the relations (22) and (23) for a given value of the time-deterministic parameter $h$, we have

$$
\begin{aligned}
f\left(S_{t}\right)= & f\left(S_{0}\right)+\int_{0}^{t} f^{\prime}\left(S_{s}\right) S_{s} a_{s} d s \\
& +\int_{0}^{t} f^{\prime}\left(S_{s}\right) S_{s-} \sigma_{s}\left(d \widetilde{W}_{s}^{h}+\vartheta_{s} d s\right)+\frac{1}{2} \int_{0}^{t} f^{\prime \prime}\left(S_{s}\right) \sigma_{s}^{2} S_{s}^{2} d s \\
& +\int_{0}^{t}\left[f\left(S_{s-}\left(1+\gamma_{s}\right)\right)-f\left(S_{s-}\right)\right]\left(d \widetilde{M}_{s}^{h}+h_{s} \lambda_{s} d s\right) .
\end{aligned}
$$


If we choose $f(x)=x^{n}, n \in \mathbb{Z} \backslash\{0\}$, take the expectation with respect to the EMM $Q^{h} \in \Upsilon$, and use relation (20), we obtain

$$
\begin{aligned}
\mathbf{E}_{Q^{h}}\left[S_{t}^{n}\right]= & S_{0}^{n}+(n-1) \int_{0}^{t} \mathbf{E}^{Q^{h}}\left[S_{s}^{n}\right]\left(a_{s}-\frac{a_{s}+h_{s} \lambda_{s} \gamma_{s}}{\sigma_{s}} \sigma_{s}\right) d s \\
& +\frac{n(n-1)}{2} \int_{0}^{t} \mathbf{E}_{Q^{h}}\left[S_{s}^{n}\right] \sigma_{s}^{2} d s \\
& +\int_{0}^{t} \mathbf{E}_{Q^{h}}\left[S_{s}^{n}\right] h_{s} \lambda_{s}\left[\left(1+\gamma_{s}\right)^{n}-1\right] d s \\
= & S_{0}^{n}+\frac{n(n-1)}{2} \int_{0}^{t} \mathbf{E}_{Q^{h}}\left[S_{s}^{n}\right] \sigma_{s}^{2} d s \\
& +\int_{0}^{t} \mathbf{E}_{Q^{h}}\left[S_{s}^{n}\right] h_{s} \lambda_{s}\left[\left(1+\gamma_{s}\right)^{n}-(n-1) \gamma_{s}-1\right] d s .
\end{aligned}
$$

Since under $Q^{h}$ the price process has marginals $\left(\mu_{t}\right)$, we have

$$
\mathbf{E}_{Q^{h}}\left[S_{t}^{n}\right]=m_{t}^{(n)}:=\int_{\mathbb{R}} x^{n} \mu_{t}(d x)
$$

and then

$$
\begin{aligned}
m_{t}^{(n)}= & m_{0}^{(n)}+\frac{n(n-1)}{2} \int_{0}^{t} \sigma_{s}^{2} m_{s}^{(n)} d s \\
& +\int_{0}^{t} h_{s} \lambda_{s}\left[\left(1+\gamma_{s}\right)^{n}-(n-1) \gamma_{s}-1\right] m_{s}^{(n)} d s
\end{aligned}
$$

Thus, the application $t \mapsto m_{t}^{(n)}$ is absolutely continuous, and we can take its Radon-Nikodym derivative with respect to $t$ and, having assumed $\left(1+\gamma_{t}\right)^{n}-(n-1) \gamma_{t}-1 \neq 0$, after an easy calculation, we obtain

$$
h_{t}=\frac{\frac{d}{d t} m_{t}^{(n)}-\frac{\sigma_{t}^{2}}{2} n(n-1) m_{t}^{(n)}}{\lambda_{t}\left[\left(1+\gamma_{t}\right)^{n}-(n-1) \gamma_{t}-1\right] m_{t}^{(n)}}, \quad d t \text {-a.e., }
$$

which is well-defined since $m_{t}^{(n)}>0$. This ends the proof.

Remark 9. Actually, formula (24) implies that in order to reduce the subset $\Upsilon$ of EMMs of the model to a singleton it suffices to know, for example, the second positive moments of the price process $S$ under one of them. In this case (i.e. $n=2)$ the condition $\left(1+\gamma_{t}\right)^{n}-(n-$ 1) $\gamma_{t}-1 \neq 0$ reduces to assume $\gamma_{t} \neq 0$ for all $t \in[0, T]$. 
Remark 10. We can interpret equation (27) as a family of constraints on the moments of the marginals $\mu_{t}$ considered as unknown. Formally, if we assume that $h$ is a deterministic function of time and that every $\mu_{t}$ satisfies $\mu_{t}(d x)=g(t, x) d x$ with $g(t, x)$ sufficiently regular, from (27) and by several simple integrations by parts, it is easy to see that $g(t, x)$ must be the solution of the following EDP:

$$
\begin{aligned}
-\frac{\sigma_{t}^{2}}{2} \frac{d^{2}}{d x}\left(x^{2} g(t, x)\right) & +h_{t} \lambda_{t} \gamma_{t} \frac{d}{d x}(x g(t, x))+\frac{d}{d t} g(t, x) \\
& =h_{t} \lambda_{t} \frac{1}{1+\gamma_{t}} g\left(t, \frac{x}{1+\gamma_{t}}\right)+\left(\gamma_{t}-1\right) h_{t} \lambda_{t} g(t, x) .
\end{aligned}
$$

Remark 11. If the coefficients of the model are constant we have an even stronger conclusion: to reduce the EMMs subset $\Upsilon$ to a singleton, it suffices to know only one marginal, e.g. the terminal one $\mu_{T}$. Indeed, in this case the price process $S$ is the exponential of a Lévy process and it is well known that any finite-dimensional distributions of a Lévy process is uniquely determined by any of its (one-dimensional) marginals.

\section{Conclusions}

In this paper, given a financial market $S$, and a family of probability measures $\mathbf{M}_{N}=\left\{\mu_{t_{1}, \ldots, t_{N}}: t_{1}, \ldots, t_{N} \in \mathcal{T}\right\}$ on $\mathcal{B}\left(\mathbb{R}^{N}\right)$, we have obtained equivalent conditions for the existence and uniqueness of an EMM $Q$ such that the $Q$-distribution of every vector $\left(S_{t_{1}}, \ldots, S_{t_{N}}\right)$ is $\mu_{t_{1}, \ldots, t_{N}}$.

When the probability space is finite (Section 2), the existence of such a measure $Q$ is equivalent to the following no-arbitrage property: one cannot construct any arbitrage position by trading dynamically in the underlying and statically in a contingent claim with pdd less or equal to $N$, bought at the price given by $\mathbf{M}_{N}$. We have called this condition $N$-mixed no-arbitrage. On the other hand, $Q$ is unique in the set of all EMMs that match the set $\mathbf{M}_{N}$, if and only if each contingent claim may be replicated by a predictable trading strategy and a contingent claim with pdd less or equal to $N$.

When the probability space is arbitrary and the price of the stock is modelled by a locally bounded real-valued semimartingale (Section 3 ), we had to consider a topological notion of $N$-mixed no-arbitrage, that we have named $N$-mixed no-free-lunch. In this setting, by considering two different topologies on the set of all contingent claims, we have studied two corresponding notions of $N$-completeness. We have so established two versions of the second FTAP. 
In the last subsection, we have shown that for the Black-Scholes model with jumps, when the coefficients are deterministic and given a family of marginals for the price process, the subset of $\Upsilon$ of all EMMs induced by a parameter $h$ deterministic in time and under which the price process has exactly the pre-specified marginals reduces to a singleton.

We finally remark that our main results have an immediate generalization if, more generally, we fix the $N$-dds of the price process only on a given subset (finite or infinite) $J$ of $\mathcal{T}$. This extension is left to the reader. Future work will be devoted to investigate, with this approach, other concrete and more general examples.

\section{References}

1. Breeden, D.T. and Litzenberger, R.H. (1978): Prices of state-contingent claims implicit in option prices. J. Business 51, 621-651

2. Brigo, D. and Mercurio, F. (2000): Option pricing impact of alternative continuous-time dynamics for discretely-observed stock prices. Finance and Stochastics 4, 147-159

3. Campi, L. (2001): A weak version of Douglas Theorem with applications to finance. Prépublication n. 655 du Laboratoire de Probabilités et Modèles Aléatoires, Universités de Paris VI et VII.

4. Carr, P., H. Geman, H., Madan, D.B. and Yor, M. (2003): Stochastic volatility for Lévy processes. Math. Finance 13(3), 345-382.

5. Delbaen, F. and Schachermayer, W. (1994): A general version of the fundamental theorem of asset pricing. Math. Annalen, Vol. 300, 463-520

6. Delbaen, F. and Schachermayer, W. (1996): The variance-optimal martingale measure for continuous processes. Bernoulli 2, 81-105

7. Douglas, R.G. (1964): On extremal measures and subspace density. Michigan Math. J. 11, 644-652

8. Dupire, B. (1997): Pricing and Hedging with Smiles. In: Dempster, M.A.H, and Pliska, S.R., (eds) Mathematics of Derivative Securities. Cambridge University Press, Cambridge

9. Föllmer, H. and Schied, A. (2002). Stochastic Finance: an Introduction in Discrete Time, de Gruyter Studies in Mathematics 27. De Gruyter, Berlin-New York.

10. Föllmer, H. and Schweizer, M. (1991): Hedging of contingent claims under incomplete information. In: Davis, M.H.A., Elliot, R.J., (eds) Applied Stochastic Analysis, Stochastics Monograph 5. Gordon Breach, London-New York, pp. 389-414

11. Föllmer, H., Wu, C.-T., and Yor, M. (2000): On Weak Brownian Motions of arbitrary order. Annales de l'Institut H. Poincaré 36, 447-478

12. Frittelli, M. (2000): The minimal entropy martingale measure and the valuation problem in incomplete markets. Math. Finance 10, 39-52

13. Goll, T. and Ruschendorf, L. (2002): Minimal distance martingale measures and optimal portfolios consistent with observed market prices. In: Buckdahn, R., Engelbert, H.J., and Yor, M. (eds), Winter School of Stochastic Processes and related topics, Stochastics Monographs 
14. Harrison, J.M. and Pliska, S.R. (1981): Martingales and Stochastic Integrals in the Theory of Continuous Trading. Stochastic Processes and their Applications 11, 215-260

15. Henderson, V. and Hobson, D.G. (2001): Coupling and Option Price Comparisons in a Jump Diffusion Model. Preprint, downloadable at http://www.bath.ac.uk/ masdgh/publications.html.

16. Hobson, D.G. (1998): Robust hedging of the lookback option. Finance and Stochastics 2, 329-347

17. Kellerer, H.G. (1972): Markov-Komposition und eine Anwendung auf Martingale. Math. Ann. 198, 99-122

18. Madan, D.B. and M. Yor (2002): Making Markov martingales meet marginals with explicit constructions. Bernoulli 8, 509-536.

19. Merton, R.C. (1976): Option pricing when underlying stock returns are discontinuous. Journal of Financial Economics 3, 125-144.

20. Miyahara, Y. (1996): Canonical martingale measures of incomplete assets markets; in Probability Theory and mathematical statistics (Tokyo, 1995), 343352. River Edge, New York: World Sci. Publishing.

21. Naimark, M.A. (1947): Extremal spectral functions of a symmetric operator. Bull. Acad. Sci. URSS Sér. Math. 11, 327-344.

22. Peccati, G. (2003): Explicit formulae for time-space Brownian chaos. Bernoulli 9 (1), 25-48.

23. Runggaldier, W. J. (2003): Jump-Diffusion models. In: Rachev, S.T.(ed.), Handbook of Heavy Tailed Distributions in Finance, North Holland Handbooks in Finance, Book 1 (W.Ziemba Series Ed.). Elsevier/North-Holland

24. Schachermayer, W. (200): Introduction to the Mathematics of Financial Markets. In: Lectures on probability theory and statistics, Saint-Flour 2000, Lecture Notes in Math., 1816. Springer Verlag.

25. Strassen, V. (1965): The existence of probability measures with given marginals. Ann. Math. Stat. 36, 423-439

26. Thierbach, F. (2002): Mean-Variance Hedging under Additional Market Information. Preprint, University of Bonn. 